\title{
QUASAR VARIABILITY FROM MICROLENSING
}

\author{
M.R.S. HAWKINS \\ Royal Observatory \\ Blackford Hill \\ Edinburgh EH9 зHJ
}

Quasars are known to vary in brightness over a wide range of time scales. Short term intrinsic variability has been well documented, and a strong case can be made that long term variation is due to microlensing. In this paper the effect of time dilation as a means for distinguishing between intrinsic variation and gravitational lensing is discussed.

The idea that small compact bodies might cause quasars to vary has been examined in some detail over the last few years, and extensive numerical simulations have been carried out to explore the effect (Kayser et al. 1986, Schneider \& Weiss 1987). Hawkins (1993) suggested that in fact all quasars are being microlensed, and that this is the main mechanism for large amplitude long term variation. Press \& Gunn (1973) in a classic paper pointed out that if every line of sight is gravitationally lensed then the mass density of the lensing bodies must be approximately equal to the cosmological critical density (i.e. $\Omega_{l e n s} \approx 1$ ). This clearly has important consequences for the dark matter problem, as well as throwing interesting new light on the behavior of quasars.

The question of whether quasar variability is caused by microlensing is explored in more detail in a more recent paper by Hawkins (1995). The point to be decided is the extent to which intrinsic variability in quasars is confined to the well established, short term, small amplitude fluctuations, and whether it can account for the large amplitude long time scale variations which dominate quasar light curves.

Among the arguments used to support the microlensing hypothesis is the apparent lack of a time dilation effect in quasar light curves. One would expect to see a lengthening of the time scale of quasar variation with redshift by a factor $(1+z)$ if the variation is intrinsic to the quasar. Many attempts have been made to detect this effect but there have been no convincing measurements of it to date. Recently Baganoff \& Malkan (1995) 
advanced an ingenious argument to circumvent this difficulty, which raises some interesting questions about the nature of quasar variability.

The main point of Baganoff \& Malkan's argument is that at higher redshift the observer is looking progressively further into the ultra-violet, and hence sees an emission region which may well be smaller than at larger wavelengths. They then put forward a model to link the decreasing size of the continuum region with shorter time scale of variability, and claim that this just cancels out the time dilation effect.

The model requires that the time scale of variation in AGN's gets shorter as the wavelength of observation decreases. This prediction can be tested in various ways. The Seyfert galaxy NGC 5548 has been closely monitored in several major observing campaigns with IUE. Peterson et al. (1991) give light curves in the optical at $4870 \AA$ and the ultra-violet at $1350 \AA$. The correlation between the light curves is almost exact, with only a difference in amplitude. There is clearly no question of a difference in time scales. A similar effect can be seen in the data of Hawkins (1995) where the time varying autocorrelation function (ACF) is evaluated for samples of low and high redshift quasars. For each sample, ACF's for blue and red passbands are shown. Although in each case a small decrease in time scale is seen from low to high redshift, in each redshift bin the time scales for the red and blue passbands are not significantly different. A similar manifestation of this may be seen in the light curves in Hawkins (1995) where the variation is achromatic.

Lack of a time dilation effect thus continues to be an argument against long term intrinsic variability in quasars. There are other aspects of the light curves such as statistical symmetry and achromatic variation which further constrain any model of intrinsic variability.

\section{References}

Baganoff, F.K. \& Malkan M.A., 1995, ApJL, 444, L13

Hawkins, M.R.S., 1990, Nature, 366, 242

Hawkins, M.R.S., 1995, MNRAS, in press

Kayser, R., Refsdal, S. \& Stabell, R., 1986, A\&A, 166, 36

Peterson, B.M., et al., 1991, ApJ, 368, 119

Press, W.H. \& Gunn, J.E., 1973, ApJ, 185, 397

Schneider, P. \& Weiss, A., 1987, A\&A, 171, 49 